\title{
Integrated and Intelligent Safety and Security System for Digital Red Light Offenders and Automatic Ambulance Rescue System Using GSM Technology
}

\author{
Bhawna Rai ${ }^{1}$, Vivek Chawla ${ }^{2}$ \\ ${ }^{1}$ M-tech, Robotics and Automation, Indira Gandhi Delhi Technical University for Women (IGDTUW), Delhi, India \\ ${ }^{2}$ Assistant Professor, Department of Mechanical and Automation Engineering, Indira Gandhi Delhi Technical University for Women \\ (IGDTUW), Delhi, India
}

\begin{abstract}
In the modern cities, as the population increases, vehicular traffic is also increasing and this leads to congestion problem, which is becoming the major issues in all over the world. Due to this, traffic management becoming the most important problem and this management fails when an accident occur. At current years, many traffic accidents occur, due to the collision of vehicles and this is because of red light jumper, who crosses the road when red light is on. To solve this problem traffic police is used but they have not been much effective. To overcome this problem we have used RF module, which is used to detects those vehicles which jumps he red light traffic signals and with it use a GSM module which is used for send the message to vehicle owner as well as next crossing traffic police. As well as this system also works to rescue ambulance which is trapped in the jam. This system will provide the ambulance a jam freepath.
\end{abstract}

Keywords: GSM Module, RF Module, Arduino UNO, Traffic light

\section{Introduction}

We know that there is no sound system which correctly detects all vehicles who jumps the red light traffic signals at India. Traffic police force is used for this purpose but they have not been much effective besides of this the matter of corruption and bribery which allows the vehicle drivers to break law freely. This project is about to overcome all these problems and detection of all those vehicles that jumps the red light and due to this accident occurred as well as this system is also work for ambulance rescue operation. Most accidents occur due to speeding and jumping red lights, but the fine for either offence a paltry Rs 100 is no deterrent for rash drivers. Proposal to increase the penalty for these offences are stuck in red tape. Traffic experts say that if the traffic challans has increased there will be a chance to stop this kind of act. Traffic congestion is also creating a major problem in modern urban areas, which have mostly creates a problem for ambulances. Many deaths occur while facing the congestion in roads.

Thus we propose a new design for automatically controlling the traffic light signal as well as work for ambulance rescue operation and achieving the above mentioned tasks so that ambulance will be able to cross all the junctions without waiting. For this here we used an RF module which is a small electronic circuit, used for transmit and receive the radio waves on one of a number of carrier frequencies. Here $\mathrm{RF}$ module is applied to a placed at traffic signals. Road marking is done according to required range. Now any vehicle crossing that road marking while red light is on will be detected from module. After this GSM module automatically send the message (fine) to the vehicle owner as well as to the traffic police of next crossing.

Corresponding data is fetched from central database using details of detected RF module. With it this system automatically send the message to the ambulance if jam is occur in particular lane due to this ambulance took some other path and not stuck in the jam. Hence we can say that this project will help in resolving problem of detection of red light jumper and reduce accident problem and also rescue the ambulance.

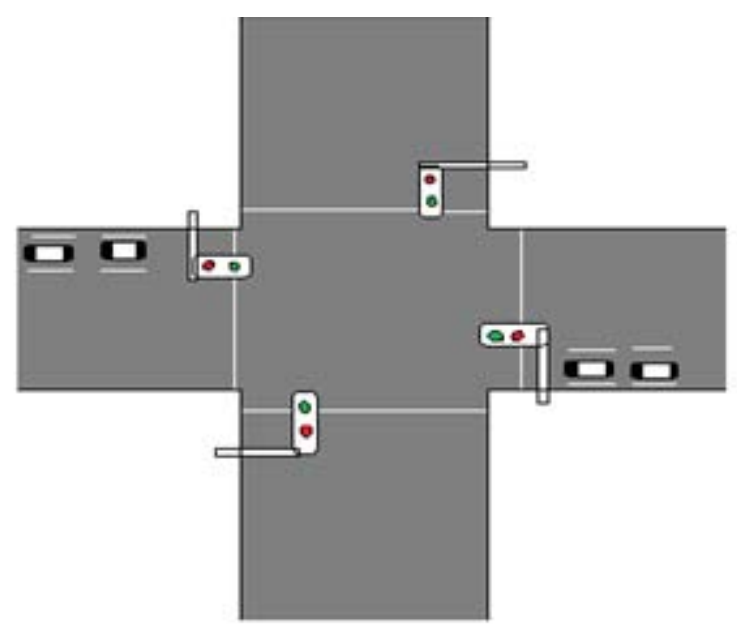

Figure 1: Red light traffic system

If any vehicles cutting the queues at crossing solid white lines on the road can slow down the traffic flow and causes traffic congestion and accidents. With it if we are able to reduce this problem we can also save many lives by giving the correct path to the ambulance which is rushed free.

\section{Analysis}

Volume 5 Issue 4, April 2016 www.ijsr.net 


\section{International Journal of Science and Research (IJSR) \\ ISSN (Online): 2319-7064 \\ Index Copernicus Value (2013): 6.14 | Impact Factor (2015): 6.391}

\subsection{Statistics}

Year 2015, 3.3 lakh challans have been issued for jumping red lights and 39,445 for speeding till May 15. Corresponding data for speeding is not available as the echallan system was introduced only in the later part of last year.

According to the National Crime Records Bureau (NCRB), Speeding and dangerous driving were the biggest reason for road fatalities. According to the WHO statistics in 2002, out of about 11.8 lakh road accident death across the world, 84,674 deaths were reported from India alone. In the year 2004, the number of road accident deaths in India increased to 92,618 .

If we compare the death ratio of India and China, we can see that India's death rate continuously increased as compare to China.

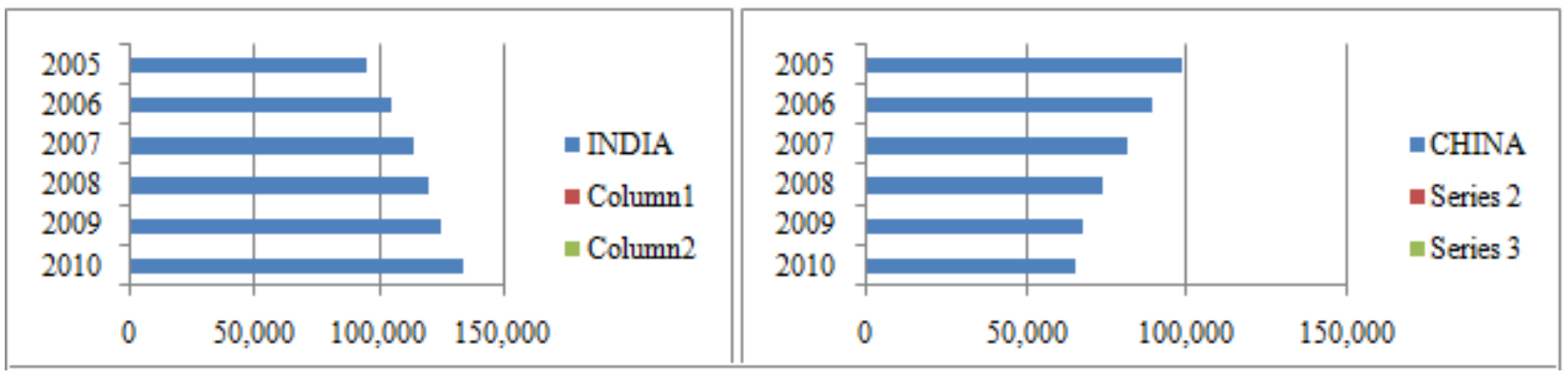

Figure 2: Road Accident Deaths India and China, 2005-10

To overcome all these problem using new technique i.e. GSM operated safety and security system for automatically operated digital red light jumper as well as for ambulance rescue operation

\section{Literature Review}

In [1] Kale, Sarika Baburao, and Gajanan P. Dhok gave a dissusion on traffic light controller and that will minimize the waiting time of vehicle. This system work in heavy traffic and set the priority of emergency vehicles. In [2] Athavan, $\mathrm{K}$ gave a scheme AARS (Automatic rescue ambulance system). The main purpose of this scheme is to provide a smooth flow for the ambulance to reach the hospital within the time. In [3] Marikhu, Ramesh works on image processing concept to reduce road accident. In [4] Assum, Terje focus on the road lightning due to this reduce the risk of accidents. In [5] Kamal, Md Abdus Samad gave the concept to reduce the congestion so that smooth flow of traffic achieve. In [6] Wei, Wang, and Fan Hanbo gave the concept of an automatic alarm device which automatic detect any accidents, search the location and sends the basic information to the first aid so that help will provide at time. In [7] Papageorgiou, Markos, et al introduce the control of congestion problem via uses different type of control strategies. In [8] Chowdhury, Tandrima, Smriti Singh, and S. Maflin Shaby is also gave the concept to pass the vehicle like ambulance according to the priority wise using Automatic rescue ambulance system (AARS) scheme. In [9] Fisher, P. David gave the concept of radar which are used to help for offenders by using laser, cameras etc.

\section{Design}

The aim of this project is to stop the road accidents which is occurred by the red light traffic jumper. In this project we show the red light jumper and detect through vehicle that jump the red light. Systems automatically check the status of red light and green light with the help of RF module. It sense automatically in case of red light and sense the information in control room. In control room system display in LCD vehicle number and it systems say the theft vehicle condition and weep the buzzer. After weeping, an automatically message generate which is send to the vehicle owner that a challan generated and one other message send to the next crossing traffic police to caught that vehicle.

For this following major components are used:

GSM Module: GSM/GPRS module is used to establish communication between a computer and a GSM-GPRS system. Global System for Mobile communication (GSM) is an architecture used for mobile communication. Global Packet Radio Service (GPRS) is an extension of GSM whichvenables higher data transmission rate. GSM/GPRS module consists of a GSM/GPRS modem assembled together with power supply circuit and communication interfaces (like RS-232, USB, etc) for computer.

RF Module: An RF module is an small electronics device used to transmit or receive radio signals between two devices. It is an embedded system, desired to communicate wirelessly between the devices. This wireless communication may be accomplished through optical communication or through RF communication.

Arduino UNO microcontroller: Arduino uno is a small computer on a single integrated circuit containing a processor, memory, external power supply, input/output peripheral, Atmega 328 microcontroller. It is easy to use because it has all the different connection at one place.

\section{Proposed Work}

Under the proposed work, fully automation is required for controlling the traffic light signal as well as work for ambulance rescue operation. All developed nation have a well developed traffic light security system with efficient traffic control on road.

In this paper, the aim is to resolve red light traffic jumping problem and rescue the ambulance using GSM module, RF 


\section{International Journal of Science and Research (IJSR) \\ ISSN (Online): 2319-7064 \\ Index Copernicus Value (2013): 6.14 | Impact Factor (2015): 6.391}

module, Arduino UNO. This system can do the following: (i) Provide safety and security on the red light traffic crossing. (ii) Less chance of accident (iii) Rescue the ambulance that will be able to cross the entire junction without waiting.

This project is designed using Ardunio UNO. RF module, which are interrupted when any vehicle cross the red light signal due to this an automated message generate and send to the traffic police pre-stored mobile number via GSM module. With it an ambulance which consist of RF module, if this module can sense the red light in any particular roadside, it can follow some other path.

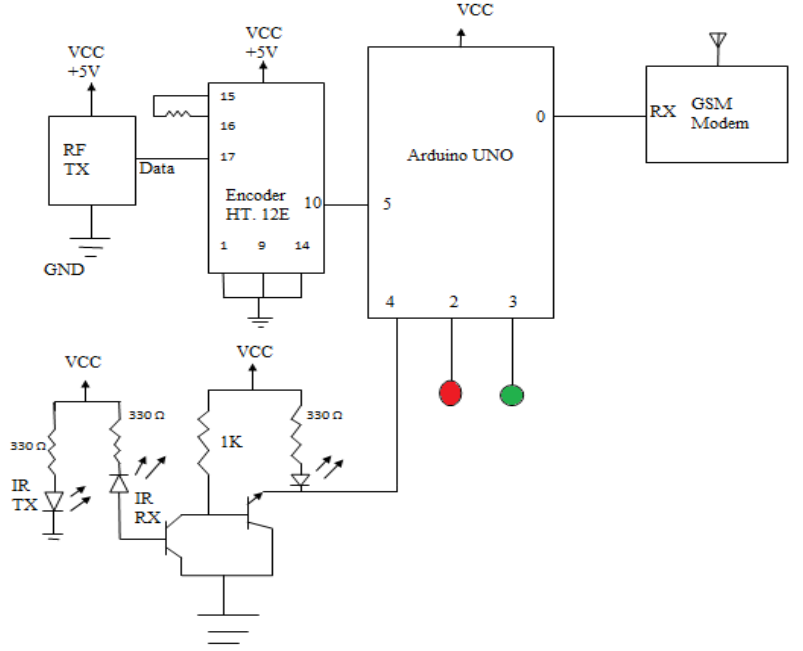

Figure 3: Connection of traffic light with GSM and control panel

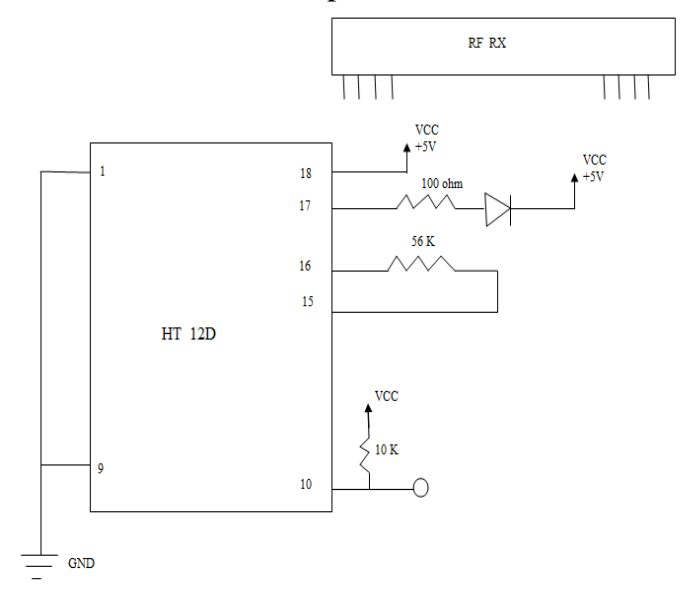

Figure 4: Circuit diagram of ambulance

\section{Conclusion}

In this paper we have studied the optimization of traffic light controllers in a City using RF module, GSM and Arduino UNO. The RF module based traffic control system works on traffic related problems such as traffic jam; unreasonable latency time of stoppage of vehicle, emergency vehicles or forcibly passing, etc. can be solved. The system has several benefits such as simple structure, high reliability, low costs, good real time, easy installation and maintenance and so on

The proposed intelligent system automatically detects red light offenders and also sends the message to the relative numbers i.e. traffic police of the next crossing. This system helps in immediate rescue of the accident and also helps the smooth flow of the traffic. As well as rescue the ambulance which got stuck into the jam and save the lives.

It can be implemented for a particular city or even an entire country.

Traffic Police department has shown genuine interest in the system. Once the system is deployed, it will prove to be beneficial to society, as it will improve traffic flow and will help reduce road accidents.

\section{References}

[1] Kale, Sarika Baburao, and Gajanan P. Dhok. "Embedded system for intelligent ambulance and traffic control management." IJCER 2.2 (2013): 137-142

[2] Athavan,K, et al. "Automatic Ambulance Rescue System. "Advanced Computing \& Communication Technologies (ACCT), 2012 Second International Conference on IEEE 2012.

[3] Marikhu Ramesh, et al. "Police Eyes: Real world automated detection of traffic violations. "Electrical Engineering/Electronics, Computer, Telecommunications and Information Technology (ECTI-CON), $201310^{\text {th }}$ International Conference on IEEE 2013

[4] AAssum, Terje, et al. "Risk compensation- the case of road lightning. "Accident Analysis \& Prevention 31.5 (1999): 545-553

[5] Kamal, Md Abdus Samad, et al. "A vehicle- intersection coordination scheme for smooth flows of traffic without using traffic lights. "Inrelligent Transportation System, IEEE Transactions on 16.3 (2015): 1136-1147

[6] Wei, Wang, and Fan Hanbo. "Traffic accident automation detection and remote alarm device. "Electric Information and Control Engineering (ICEICE), 2011 International Conference on IEEE, 2011

[7] Papageorgiou, Markos, et al. "Review of road traffic control strategies."Proceedings of the IEEE 91.12 (2003): 2043-2067.

[8] Chowdhury, Tandrima, Smriti Singh, and S. Maflin Shaby. "A Rescue System of an advanced ambulance using prioritized traffic switching. "Innovations in Information, Embedded and Communication Systems (ICIIECS), 2015 International Conference on. IEEE, 2015.

[9] Fisher, P. David. "Improving on police radar." Spectrum, IEEE 29.7 (1992): 38-43.

\section{Author Profile}

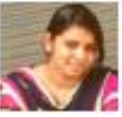

Bhawna Rai received the B.Tech degree in Electronics and Communication from Shambhunath Institute of Engineering and Technology, Allahabad, Uttar pradesh and currently persuing her M.tech from IGDTUW in Robotics and Automation branch.

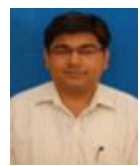

Vivek Chawla completed his M.tech from DITE Delhi, his area of specilazation are tool designing and manufacturing, CAD/CAM application and operation management. He completed his MBA from symbosis. 